\title{
Sewerage and Climate
}

\section{Khalidullin 0*}

Professor of Ecology, Academician of the Russian Federation LAN, Kazakh National University, Russia

*Corresponding Author: Khalidullin O, Professor of Ecology, Academician of the Russian Federation LAN, Kazakh National University, Russia.

Received: October 21, 2019; Published: October 29, 2019

DOI: $10.31080 /$ ASMI.2019.02.0416

The earth and atmosphere for millions of years created the animal and plant worlds. Together with them, water quality also developed. In constant motion, water formed a continuous chain of transformation in the bodies of living things and leaves of plants. The harmonious interaction of water, atmosphere and biota has led to the existing balance - the circulation of water through the atmosphere, where the properties of water acquire their initial starting quality for new transformations.

Man, in the process of industrialization, turned water into a working tool. He made the water perform unnatural functions for her. Pressure, transportation, spraying, storage, boiling, burning, poisoning of ever increasing volumes of water have led to a change in the quality of water in the atmosphere. Changes in the quality, volume and rate of evaporation across the planet lead to climate change. More details at: https://www.actascientific.com/ASMI/ pdf/ASMI-SI-01-0009.pdf

The main sources of artificial fumes are

- Reservoirs of artificial structures

- Floods - rivers overflow banks and create immense areas of evaporation.

- $\quad$ Existing crop production with deep plowing.

- Garbage and ore landfills. It is known that the area of the world landfill is equal to the area of Mexico.

- $\quad$ Aviation, and all transport destroys significant amounts of moisture in the air with the destruction of structures.

- All asphalt and concrete surfaces.

- All types of water flow for cooling, washing and everything else.

- Of particular note is water consumption in the utilities sector.
Each of the listed positions can be changed without any loss of comfort and convenience, if humanity understands the emergency of the current moment.

Considering the last position, it can be noted that toilet matters alone can significantly affect the climate. Man went against nature by mixing pure drinking water with his feces in order to transport them to one place and then clean them.

Everything that is flushed from the toilet falls into the sump. The middle and above it in the sediment represents a clear liquid. It can be used for repeated flushing from the toilet. To do this, it is necessary to disconnect the flow of water to it from the main water supply and bring the supply from the pump.

Odor can be eliminated by taking the simplest technical measures for weathering. Many different odor elimination methods are known. But the simplest and most affordable is the automatic ventilation device of the toilet itself with the release of air into the ventilation pipes. The water purified in this way is fed back to the toilet with a special pump and flushes its contents.

Thus, the small water circuit closes: toilet bowl - pipes - sludge - pipe cleaning - toilet bowl. Specialists calculated the water consumption in the household with these indicators:

https://yandex.ru/search/?text=cost $\% 20$ water\%20on $\% 201 \% 20$ person $\% 20 \mathrm{in} \% 20$ day $\&$ clid $=2270455 \&$ banerid $=6101003224 \% 3 \mathrm{~A}$ 4655801518424859060\&win=346\&lr=162: 


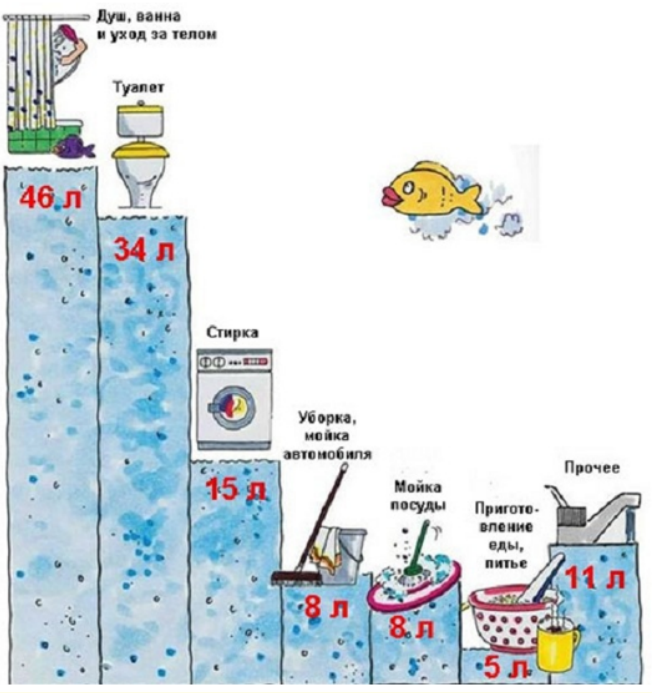

Figure 1
Thus, everything that comes into the house through the water supply system must be used $100 \%$ in place. Nothing should go into the sewers. The sewage centralization industry is disappearing. The vast territories occupied by devices and sewage enterprises with all states are being freed. But most importantly, settling tanks and reservoirs disappear - powerful sources of artificial evaporation that affect the water cycle between the atmosphere and the earth, create natural disasters and change the climate.

Volume 2 Issue 11 November 2019 (c) All rights are reserved by Khalidullin 0 .

The water in the sump is replenished with other wastewater from related consumers. If you carefully look at the costs, you can see that it can be used for other purposes, for example, for external washing of a car, sidewalks, roads. Everything else can be spent on watering green spaces near the house. You can accumulate water in special tanks, from several houses, gradually treat it by smell and use it for washing roads, for a fire reserve.

In addition, knowing how water is used in spaceships, it is not difficult to pick up a purification complex and use this water to wash everything else, except dishes and drink. In any case, one must be prepared for the fact that the flow of clean water will disappear. This is already happening in some places, for example, in South Africa, Australia.

It will be necessary to create devices a little more complicated for the treatment of water near each house or area. The separated solid waste is periodically taken to enterprises for incineration and / or preparation of fertilizers. 